\title{
4
}

\section{Digital Holographic Interferometric Characterization of Optical Waveguides}

\author{
Hamdy Wahba and Mamdouh Shams El-Din \\ Physics Department, Faculty of Science, 34517 Damietta, \\ University of Mansoura \\ Egypt
}

\section{Introduction}

Holography was developed by Dennis Gabor 1947. Gabor presented holography as a lensless process for image formation by reconstructed wavefronts (Gabor, 1948, 1949, 1951). Holography can be defined as a method for recording and reconstructing whole optical wavefields, which means intensity and phase (Gabor, 1948, 1949), thus it exhibits 3D characteristics like depth of field or parallax. Holographic interferometry (Powell \& Stetson, 1965 ) is a very effective non-destructive, contactless tool to measure shape, deformation or refractive index distributions (Kreis, 1996).

In 1994 the modern digital holography was introduced (Schnars, 1994; Schnars, \& Jüptner, 1994a; Schnars, \& Jüptner, 1994b; Schnars et al., 1995; Schnars, \& Jüptner, 2005). The digital holography can be defined as digital recording of the holograms and the numerical reconstruction of the wave fields in a computer, where, the charge coupled devices (CCDs) are the most frequently used devices to record the holograms. The digital holography in the last ten years was involved in a lot of applications due to the development of powerful computers, ultra large memories and smaller pixel size CCD targets (Kreis, 2005).

The phase shifting interferometric (PSI) technique was introduced by Hariharan et al. into the field of holography as an accurate method for real time fringe measurement (Hariharan et al., 1982). Furthermore, PSI combined with digital holography (Skarman et al., 1996). Yamaguchi and Zhang 1997 improved phase shifting digital holography (PSDH) (Yamaguchi \& Zhang, 1997). The phase difference using PSDH is measured with an accuracy of $2 \pi / 200$ (Hariharan, 2002).

For a long time, the determination of refractive index distributions in fibres, optical waveguides or other transparent solids was performed by interferometric methods. Twobeam and multiple beam based interferometers were used as a non-destructive tool to determine the optical parameters of fibres (Faust, 1952, 1954; Marhic et al., 1975). While, the mathematics used in (Marhic et al., 1975; Saunder \& Gardner, 1977; Barakat et al., 1985) neglect the non-straightforward refraction of the light beam inside the fibres. Hamza et al. (Hamza et al., 1994, 1995) constructed an accurate mathematical model (multilayer model) which considered the exact local refraction of the incident beam on its way through the graded index optical fibre, which is divided into a large number of thin concentric layers of constant refractive index. This model was verified with two-beam and multiple-beam interferometers. The consideration of incident beam refraction gave a better accuracy in the 
determination of the optical parameters of graded index optical fibres (Hamza et al., 1995, 2001), thick optical fibres (Hamza et al., 2004) and mechanically stressed optical fibres (Sokkar et al., 2008) than without this consideration. Automated Fizeau interferometric techniques were applied in studies of the optical and optothermal properties of fibres (ElMorsy et al., 2002a, 2002b; Hamza et al., 2007). These cited developments substantially increased the accuracy of the measured optical fibre parameters.

In addition, digital holographic interferometry (DHI) is used for determining refractive index profiles. Digital holographic microscopy (DHM) was used to measure the mean integral refractive index and thickness of living cells (Rappaz et al., 2005). The absolute accuracy of the mean refractive index measurement was about 0.0003. Also, Kemper et al. (Kemper \& Carl, 2006; Kemper et al., 2007) used DHI to measure the refractive index and thickness of living cells. Kebbel et al. applied digital holography to refractive index variations within transparent media in microgravity experiments (Kebbel et al., 1999). Two-dimensional refractive index profiles of phase gratings have been investigated using DHI (De Angelis et al., 2006) as well as the refractive indices of liquids using lensless Fourier DHI (Hossain et al., 2006). The high performance of DHI was tested by measurements of low variation refractive indices of fluids in a comparative study with other techniques (Dubois et al., 1999; Owen \& Zozulya, 2002) like traditional MachZehnder interferometry. The coupling of digital holographic microscopy and polarization imaging digital holography was demonstrated in an investigation of induced birefringence in non striped bent optical fibres and the birefringence of stressed PMMA (Cuche et al., 1999; Colomb et al., 2002, 2005). The mathematics describing the refractive index of transparent materials used in (Dubois et al., 1999; Owen \& Zozulya, 2002; De Angelis et al., 2006; Hossain et al., 2006) cannot be used directly to measure and configure the refractive index profile of GRIN optical waveguides, since it assumes a constant refractive index along the light path in a material. So it can be used only to determine the mean refractive index of the GRIN optical waveguides but it does not consider the varying refraction of the beam along its path inside the fibre. Large scale strongly refracting fields produce ray bending. This effect was recognized in holographic interferometric investigations combined with iterative calculations (Sweeney \& Vest, 1973) as well as tomographic methods (Cha \& Vest, 1981).

Recently, digital holographic phase shifting interferometry with the aid of mathematical models, which consider the refraction of the incident rays, were used to investigate some optical parameters of fibrous materials (Wahba \& Kreis, 2009a, 2009b, 2009c; Yassien et al., 2010). The refractive index profile and optical parameters of graded index fibres as well as the refractive index profile of bent optical fibres were determined with accuracy $2.3 \times 10^{-4}$.

Organic or inorganic optical waveguides are made of transparent dielectric materials, e.g. graded index (GRIN) planar optical waveguides and GRIN optical fibres. The fabrication of integrated optics is a very crucial technology for optical communications and sensing devices in the near future. However, it is absolutely necessary to have accurate knowledge about the optical waveguide parameters to use it in technological applications.

In recent times, polymeric integrated optics has shifted to the focus of interest due to the low material cost and simple handing and processing of polymers during the manufacturing process. Polymethyl methacrylate (PMMA) is a very promising material to be used as a basic material to produce optical waveguides and gratings (Baker \& Dyer, 1993; Eldada \& Shacklette, 2000; Vollertsen \& Wochnowski, 2004). 
The UV-laser lithographic fabrication of integrated optical waveguides has been wellrecognized in the last years. The UV excimer laser has been used to irradiate homogeneous slabs of PMMA in order to modify the refractive index locally in a controllable way at the polymeric substrate surface (Wochnowski et al., 2000; Shams-Eldin et al., 2004, 2005). Thus the integrated optical waveguides can be directly laser-written into the surface of polymeric substrates by employing lithographic masks. It is very important that the refractive index is modified in a controllable way by using specific conditions during the irradiation process in order to obtain the desired waveguide structures (Wochnowski et al., 2000). The UV photons of the incident laser radiation do interact with the molecules of the polymeric material due to a UV-photon-induced reaction (Shams-Eldin et al., 2005), so the refractive index at the surface of the PMMA slabs was changed in the irradiated area. Consequently, the refractive index modification in the irradiated area appears as a smooth variation, which depends on the waveguide depth.

The mode field distribution across the GRIN optical waveguides is very important in applications. In the case of GRIN optical waveguides, the variation in refractive index can be presented as a function of the form of a complementary error function, exponential, Gaussian, parabolic, or shape not described by any simple mathematical function (Chiang, 1985; Mathey \& Jullien, 1996). To use such optical waveguides in telecommunication, it is useful to know the number of propagated modes and their propagation constants for any given profile. The number of modes depends on two factors; the first one is the difference between maximum and minimum refractive indices of the guiding region, the second one is the radius of the guiding region. Eguchi and Horinouchi (Eguchi \& Horinouchi, 2004) used the finite-element method to determine the number of modes of optical fibres. Shemirani et al. (Shemirani et al., 2009) developed a field-coupling model for propagation in a graded index optical fibre, analogous to the principal states model for polarization mode dispersion in the single mode fibre. This model was based on the concept of the first order principal modes, which have well defined group delays that depend on the strength of the mode coupling. This first-order model predicts a linear relationship between the intensity distributions at the input and output. This model is extended to account for higher order modal dispersion (Shemirani \& Kahn, 2009).

In this Chapter, digital holographic phase shifting interferometry is used to investigate the optical properties of graded index optical waveguides, e.g. graded index planar optical waveguides and optical fibres. A planar optical waveguide sample is prepared by the UV laser lithographic method. The approach of digital holography with the aid of phase shifting interferometry is applied. The reconstructed optical phase differences along the GRIN optical waveguides are extracted and then a simple algorithm is used to avoid the problem of tilted GRIN optical waveguides inside the optical field. The extracted optical phase differences due to the optical waveguides are aligned to be perpendicular to the $x$-axis. After this process, the mean optical phase differences across the samples are calculated and thus the errors in the calculated mean values of optical phase differences across the optical waveguides are reduced. The proposed method is used to construct the refractive index profiles across GRIN optical waveguides with the aid of the multilayer model. Also, a simple algorithm is used to reconstruct the 3D refractive index of the GRIN optical fibre considering the symmetrical distribution of the GRIN optical fibre layers. In addition, an analytical method is presented to calculate the effective indices and the mode field 
distribution across the symmetric and asymmetric GRIN optical waveguides. The digital holographic phase shifting interferometric approach affects the accuracy of the calculated parameters.

\section{Digital holographic phase shifting interferometry}

In digital holography the recorded wavefield is reconstructed by multiplying the stored hologram values by the complex conjugate of a numerical model of the reference wave $r^{*}(\zeta$, $\eta)$ and then calculating the resulting diffraction field $b^{\prime}\left(x^{\prime}, y^{\prime}\right)$ in the image plane, see Fig. 1 (Kreis, 1996, 2005; Schnars \& Jüptner, 2005). The numerical reconstruction of the digitally recorded hologram is defined as a numerical calculation of the phase and the intensity of the recorded wavefield. This is theoretically calculated by the diffraction integral;

$$
b^{\prime}\left(x^{\prime}, y^{\prime}\right)=\frac{1}{i \lambda} \int h \int(\zeta, \eta) r^{*}(\zeta, \eta) \frac{\exp \{i k \rho\}}{\rho} d \zeta d \eta
$$

with $\rho=\sqrt{d^{\prime 2}+\left(\zeta x^{\prime}\right)^{2}+\left(\eta y^{\prime}\right)^{2}}$ and $k$ is the wave number $(k=2 \pi / \lambda)$.

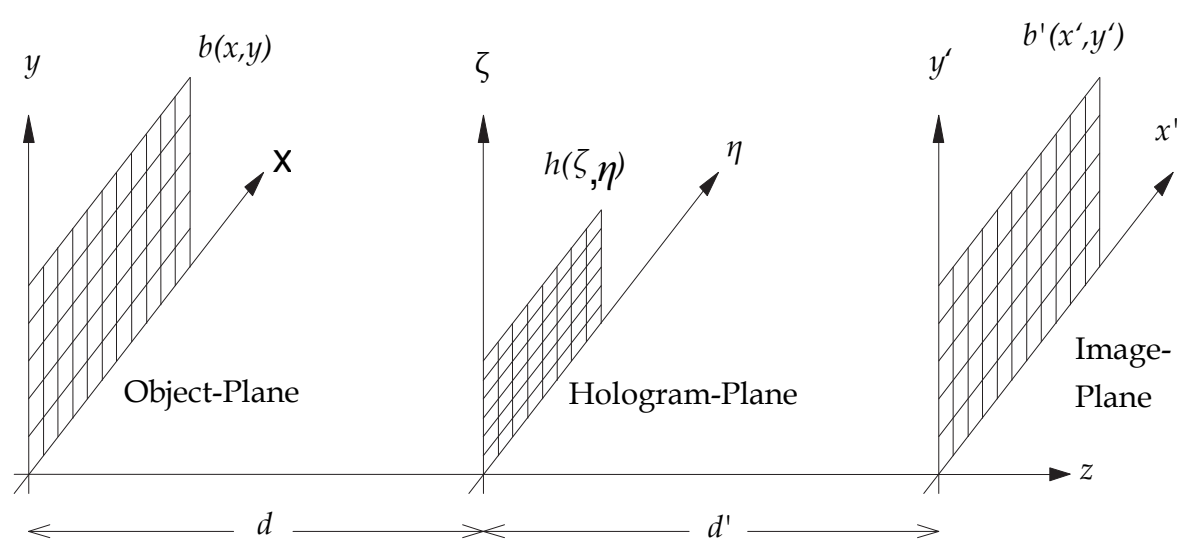

Fig. 1. Geometry of digital Fresnel holography.

The finite discrete form of the Fresnel approximation to the diffraction integral is

$$
\begin{aligned}
& b^{\prime}\left(n \Delta x^{\prime}, m \Delta y^{\prime}\right)=A \sum_{j=0}^{N} \sum_{l=}^{M} h(j \Delta \zeta, l \Delta \eta) r^{*}(j \Delta \zeta, l \Delta \eta) \\
& \quad \times \exp \left\{\frac{i \pi}{d^{\prime} \lambda}\left(j^{2} \Delta \zeta^{2}+l^{2} \Delta \eta^{2}\right)\right\} \exp \left\{2 i \pi\left(\frac{j n}{N}+\frac{l m}{M}\right)\right\} .
\end{aligned}
$$

The parameters used in this formula for calculating the complex field in the image plane are given by the CCD array used, having $N \times M$ pixels of pixel pitches $\Delta \zeta$ and $\Delta \eta$ in the two orthogonal directions. The stored hologram is $h(j \Delta \zeta, l \Delta \eta)$. The distance between the object and the CCD is denoted by $d$, and normally $d^{\prime}=d$. Complex factors not depending on the hologram under consideration are contained in a given specific CCD. 
The pixel spacing in the reconstructed field is

$$
\Delta x^{\prime}=\frac{d^{\prime} \lambda}{N \Delta \zeta} \quad \text { and } \quad \Delta y^{\prime}=\frac{d^{\prime} \lambda}{M \Delta \eta}
$$

An alternative to the Fresnel approximation uses the fact that Eq. (1) describes a convolution of $h(\zeta, \eta) r^{*}(\zeta, \eta)$, with the impulse response $g\left(x^{\prime}, y^{\prime}, \zeta, \eta\right)=(\exp \{i k \rho\}) / i \lambda \rho$. The convolution theorem states that $b^{\prime}$ is given by

$$
b^{\prime}=A^{\prime} F^{-1}\left\{F\left\{h \cdot r^{*}\right\} . F\{g\}\right\},
$$

where $F$ denotes the Fourier transform and $F^{-1}$ is its inverse. In practice, both $F$ and $F^{-1}$ are calculated by the fast Fourier transform algorithm. The resulting pixel spacing (Kreis, 2005) for this convolution approach is

$$
\Delta x^{\prime}=\Delta \zeta \quad \text { and } \quad \Delta y^{\prime}=\Delta \eta
$$

The use of a real hologram in the Fresnel reconstruction or the convolution reconstruction, leads to a strong d.c. term, a focused real image, and a virtual image that is not sharp. The complex field can be recorded and calculated by phase-shifting digital holography. The calculated complex wavefield is used instead of a real hologram in the convolution approach to overcome the problems of the d.c. term and twin image. For this purpose several holograms, at least three, with known mutual phase shifts are recorded. These holograms are given by

$$
I_{n}=a(\zeta, \eta)+b(\zeta, \eta) \cos \left(\varphi(\zeta, \eta)+\varphi_{R n}\right), \quad n=1,2,3, \ldots \ldots
$$

where $a(\zeta, \eta)$ and $b(\zeta, \eta)$ are the additive and the multiplicative distortions and $\varphi_{R n}$ is the phase shift performed in the reference wave during recording of the holograms. In our case the phase shift is $90^{\circ}$, and it starts with $\varphi_{R n}=0^{\circ}$. In this case we get a set of four linear equations that are point wise solved by a Gaussian least squares method(Kreis, 1996). The complex wavefield in the hologram plane can be calculated from

$$
H(\zeta, \eta)=\left[I_{1}(\zeta, \eta)-I_{3}(\zeta, \eta)\right]+i\left[I_{4}(\zeta, \eta)-I_{2}(\zeta, \eta)\right]
$$

Finally, the reconstruction process is based on the use of the complex wavefield in the convolution algorithm. The intensity distribution in the reconstruction plane is given by

$$
I\left(x^{\prime}, y^{\prime}\right)=\left|b^{\prime}\left(x^{\prime}, y^{\prime}\right)\right|^{2}
$$

and the phase distribution is given by

$$
\varphi\left(x^{\prime}, y^{\prime}\right)=\arctan \left\{\frac{\operatorname{Im}\left|b^{\prime}\left(x^{\prime}, y^{\prime}\right)\right|}{\operatorname{Re}\left|b^{\prime}\left(x^{\prime}, y^{\prime}\right)\right|}\right\}
$$

Then, the optical phase difference due to the used phase object such as GRIN optical waveguides can be extracted. 


\section{Refractive index profile of GRIN optical fibre using the multilayer model}

The GRIN optical fibre sample can be considered as a phase object. When it is implemented in the path of one of the interfering beams of a Mach-Zehnder interferometer, the produced fringes suffer shifts in the GRIN optical fibre region. These shifts represent the optical path difference between the light beam traversing the fibre and that traversing the surrounding medium.

In GRIN optical fibre, the core is surrounded by a homogeneous cladding of refractive index $n_{c l}$. In the multilayer model, the core and cladding of the graded index optical fibre are assumed to consist of a large number of thin layers. Each layer has an annular cross section and is considered to have a thickness $a$ and refractive index $n_{j}$ where the layers are numbered by $j=1, \ldots, N$ with $N=R / a$. $R$ is the core radius. If this optical fibre is immersed in a liquid of refractive index $n_{L}$, there will be a refraction of the incident beam at the boundary between the liquid and the cladding. Hamza et al. obtained a recurrence relation which predicts the optical path of the refracted beam through $Q$ layers of the fibre. The GRIN fibre is illuminated by a collimated beam, with a ray crossing the centre of the core defining the optical axis (Hamza et al., 1995). An arbitrary beam transverses the fibre at a distance $d_{Q}$ from the optical axis and leaves the fibre at a distance $x_{Q}$. Assume a coordinate system whose origin is at the fibre centre, then the corresponding fringe shift is defined as $Z_{Q}$ and the optical path difference $\delta_{Q}$ is given by the recurrence formula

$$
\begin{aligned}
\frac{\lambda Z_{Q}}{h}= & \frac{\lambda}{2 \pi} \Delta \phi_{Q}=\sum_{j=1}^{Q} 2 n_{j}\left(\sqrt{(R-(j-1) a)^{2}-d_{Q}^{2} n_{L}^{2} / n_{j}^{2}}-\sqrt{(R-j a)^{2}-d_{Q}^{2} n_{L}^{2} / n_{j}^{2}}\right), \\
& +2 n_{Q}\left(\sqrt{(R-(Q-1) a)^{2}-d_{Q}^{2} n_{L}^{2} / n_{Q}^{2}}\right)-n_{L}\left(\sqrt{R^{2}-d_{Q}^{2}}+\sqrt{R^{2}-x_{Q}^{2}}\right)
\end{aligned}
$$

with $Q$ running from 1 to $N, h$ is the interfringe spacing and $\Delta \varphi_{Q}$ is the interference phase difference.

This recurrence relation also describes the shape of the fringes which is produced as a result of the refraction of the incident beam through the $Q$ layers of the fibre. Without loss of generality, we can assume that the incident light beam passes through the middle of the Qth layer. Thus the value of $d_{Q}$ in Eq. (10) can be obtained from the following relation

$$
d_{Q}=\frac{n[R-(Q-0.5) a]}{n_{L}}
$$

Eq. (10) can now be used to get the refractive index profile for GRIN optical fibres $(N \rightarrow \infty)$. Hamza et al. have shown that inhomogeneous refraction must be considered in the measurement and calculation of optical fibre parameters, so that the accuracy of the measured parameters of GRIN optical fibre is increased (Hamza et al., 1994, 1995).

\section{Characterization of TE modes in the GRIN optical waveguides}

The study of the GRIN optical waveguides using the wave equation to achieve the allowed propagating modes is familiar from basic quantum mechanics. The analogy between the particle in box and optical waveguide problem is very strong: both situations describe waves, which are confined between two reflecting boundaries. In both cases, the waves 
partially tunnel into the surrounding potential barrier before turning around. Only certain allowed energies (in the case of the particle), or propagation coefficients $\beta$ (in the case of the optical waveguide) create a standing wave in one-dimensional system.

\subsection{Symmetric optical waveguide}

The squared value of the refractive index distribution at the core of the GRIN optical waveguide is shown in Fig. 2. At the centre of the core the refractive index has a maximum value $n_{0}$ and then decreases to reach $n_{\text {clad }}$ at $x= \pm R$ ( $R$ is the radius of the core). The squared value of the refractive index distribution is given by,

$$
n^{2}(x)=\left\{\begin{array}{ll}
n_{\text {clad }}^{2}, & -R>x, R<x \\
\left(n_{o}-\Delta n\left(\frac{x}{R}\right)^{2}\right)^{2}, & -R<x>R
\end{array}\right\}
$$

where $\Delta n=n_{0}-n_{\text {clad }}$.

The guided wave problem requires only the knowledge of the square of the refractive index distribution.

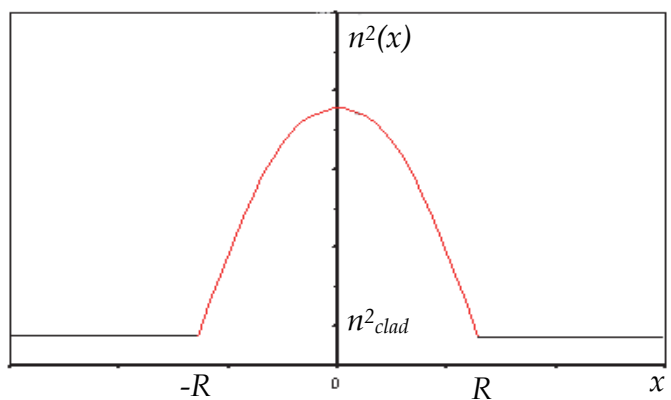

Fig. 2. The core of the GRIN optical fibre is confined between cladding media, arranged such that the squared refractive index $n^{2}(x)$ is larger than that of the surrounding media $\left(n_{\text {clad }}^{2}\right)$.

In the TE case, the E field is polarized along the $y$ - axis and propagating along the $z$-axis. The TE modes of the GRIN optical waveguide are obtained as the solutions of the following equations (Conwell, 1973; Marcuse, 1973),

$$
\begin{gathered}
\frac{d^{2} E_{y}}{d x^{2}}+\left(k_{o}^{2} n^{2}(x)-\beta^{2}\right) E_{y}=0, \\
-\frac{i}{w \mu_{o}} \frac{\partial E_{y}}{\partial z}=H_{x}, \\
\frac{i}{w \mu_{o}} \frac{\partial E_{y}}{\partial x}=H_{z},
\end{gathered}
$$


where

$$
k_{o}^{2}=\omega^{2} \varepsilon_{o} \mu_{o}
$$

$k_{0}, \mu_{0}, \omega$ and $\beta$ are the wave number, the permeability constant, the angular frequency and the allowed propagation coefficient along the $z$-axis, respectively.

Applying the continuity boundary conditions that connect the solutions at the interfaces $x=$ $R$ and $x=-R$ and for $\Delta n<<n_{0}$, the solutions of the Eqs. (13)-(15) in the three regions (symmetric waveguide) are given in terms of Hermite polynomials $\left(H_{q}\right)$ of degree $q$, as

$$
E_{y}(x)=\left\{\begin{array}{l}
A e^{-\frac{k_{o}(\Delta \varepsilon)^{\frac{1}{2}} R}{2}} H_{q}\left(R \sqrt{\frac{k_{o}(\Delta \varepsilon)^{\frac{1}{2}}}{2 R}}\right) e^{-\gamma(x-R)}, x>R \\
A e^{-\frac{k_{o}(\Delta \varepsilon)^{\frac{1}{2}} x^{2}}{2 R}} H_{q}\left(\sqrt{\frac{k_{o}(\Delta \varepsilon)^{\frac{1}{2}}}{2 R}} x\right),-R<x<R \\
A e^{-\frac{k_{o}(\Delta \varepsilon)^{\frac{1}{2}} R}{2}} H_{q}\left(-R \sqrt{\frac{k_{o}(\Delta \varepsilon)^{\frac{1}{2}}}{2 R}}\right) e^{\gamma(x+R)}, x<-R
\end{array}\right\}
$$

where $q$ is an integer that identifies the mode. $H_{q}$ is the appropriate Hermite polynomial defined by

$$
H_{q}(x)=(-1)^{q} e^{x^{2}} \frac{d^{q}}{d x^{q}} e^{-x^{2}}
$$

The first three Hermite polynomials in $x$ are

$$
\begin{aligned}
& H_{0}(x)=1 \\
& H_{1}(x)=2 x \\
& H_{2}(x)=4 x^{2}-2
\end{aligned}
$$

Also $\gamma$ in Eq. (17) is the attenuation coefficient in the clad region and $\Delta \varepsilon=2 n_{0} \Delta n$. The allowed propagation coefficient and the effective index for every mode are given respectively by

$$
\beta=\sqrt{k_{o}^{2} \varepsilon_{o}-\frac{k_{o} \sqrt{\Delta \varepsilon}}{R}(1+2 q)}
$$

and

$$
n_{e f f}=\frac{\beta}{k_{o}}
$$

where $\varepsilon_{0}$ is the dielectric constant at the center of the symmetric optical waveguide, and it is related to the refractive index by $\varepsilon_{o}=n_{o}^{2}$. 


\subsection{Asymmetric optical waveguide}

On the other hand, to find the allowed TE modes and the propagation coefficients of the asymmetric GRIN optical waveguide, we assume that, the GRIN optical waveguide, as shown in Fig. 3, consists of two graded index zones; both have a Gaussian refractive index profile. The first zone is in the range $0<x<b_{1}$, and the second one is in the range $b_{2}<x<b_{4}$. There is a very thin layer of constant refractive index $n_{s 1}$ confined between the two zones in the range $b_{1}<x<b_{2}$. The plane at $x=0$ represents the interface between the surface of the first zone of the optical waveguide and the immersion liquid (of refractive index $n_{L}$ ) and the plane at $x=b_{4}$ represents the interface between the second zone of the waveguide and the substrate, which has refractive index $n_{s}$.

\subsubsection{TE modes in the first zone of waveguide}

The refractive index profile for each zone is fitted using a Gaussian function which could be used to determine the allowed TE modes for the optical waveguide sample. The first zone of the waveguide in the range $0<x<b_{1}$ is represented by the Gaussian refractive index profile given by

$$
n(x)=n_{s_{1}}+\Delta n_{1} e^{-\left(\frac{x}{b}\right)^{2}} \quad, \quad 0<x<b_{1}
$$

where $b$ is the depth of the first zone (between 0 and $b_{1}$ ), and $\Delta n_{1}$ is the difference between the refractive indices at $x=0$ and $x=b_{1}$. The conditions for the wave propagation along the first zone of the waveguide are: (1) a zigzag of the beam path and (2) the total phase change must be a multiple of $2 \pi$. Using WKB approximation (Marcuse, 1973; Mathey, 1996), the lateral resonance condition for the first zone is given by

$$
2 \int_{0}^{b_{1}} \sqrt{k_{o}^{2} n^{2}(x)-\beta^{2}} d x-2 \Phi_{1 / 2}-2 \Phi_{1 / 3}=2 m \pi, \quad 1 \leq m \leq M
$$

where $\beta=k_{o} n_{\text {eff }}$ is the propagation constant of the $m^{\text {th }}$ mode; $m$ is an integer. $M$ is the total number of modes, $k_{0}=2 \pi / \lambda$ is the wave-vector, $\lambda$ is the wavelength, and $n_{\text {eff }}$ is the effective

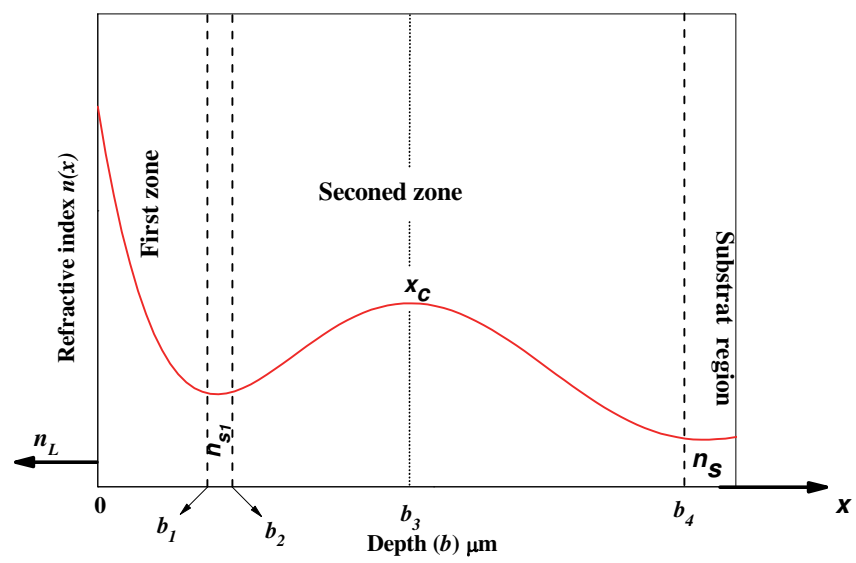

Fig. 3. The waveguide confined between liquid and substrate media, arranged such that the refractive index $n(x)$ is larger than that of the surrounding media $\left(n_{L}\right)$ and $\left(n_{s}\right)$. 
mode index for light that propagates along the optical waveguide. $\Phi_{1 / 2}$ and $\Phi_{1 / 3}$ are the phase changes at the film-liquid interface and at the first and second zones interface, respectively. Generally these last quantities are approximated to $\Phi_{1 / 2} \approx \pi / 2$ and $\Phi_{1 / 3} \approx \pi / 4$ (Mathey, 1996). The effective indices $\left(n_{\text {eff }}\right)$ for the first zone are obtained numerically by substituting Eq. (22) into Eq. (23).

\subsubsection{TE modes of second zone waveguide}

Referring to Fig. 3, we suppose that, without loss of generality, the second region of the waveguide in the range $b_{2}<x<b_{4}$ is divided into two asymmetric parts which have an individually Gaussian refractive index profile. The first and the second parts within the ranges are $b_{2}<x<b_{3}$ and $b_{3}<x<b_{4}$, respectively. The refractive index profile in these two parts of the second zone of the waveguide is given by

$$
n(x)=\left\{\begin{array}{ll}
n_{s_{1}}+\Delta n_{2} e^{-\left(\frac{x-x_{c}}{b}\right)^{2}} & , b_{2}<x<b_{3} \\
n_{s_{2}}+\Delta n_{3} e^{-\left(\frac{x-x_{c}}{b}\right)^{2}} & , b_{3}<x<b_{4}
\end{array},\right.
$$

where $b$ is the irradiation depth for both, the first part between $b_{2}$ and $b_{3}$, and the second part between $b_{3}$ and $b_{4} . \Delta n_{2}$ is the difference between the refractive indices at the interface between the first and the second parts at $x=b_{3}$ and that at $x=b_{2}$, whereas $\Delta n_{3}$ is the difference between the refractive indices at the interface between the first and the second parts at $x=b 3$ and that of the substrate at $x=b 4$.

Similarly, by analogy with Eq. (23) we have for the first and second parts

$$
\begin{array}{ll}
2 \int_{b_{2}}^{b_{3}} \sqrt{k_{o}^{2} n^{2}(x)-\beta^{2}} d x-2 \Phi_{1 / 3}=2 m \pi, & 1 \leq m \leq M \\
2 \int_{b_{3}}^{b_{4}} \sqrt{k_{o}^{2} n^{2}(x)-\beta^{2}} d x-2 \Phi_{1 / 3}=2 m \pi, & 1 \leq m \leq M
\end{array}
$$

since $\Phi_{1 / 2}=0$ at $x_{c}$.

The effective indices $\left(n_{\text {eff }}\right)$ for the two parts of the second zone are obtained numerically by using Eq. (24) in Eqs. (25) and (26).

\section{Digital holographic set-up}

The digital holographic setup is a Mach-Zehnder system, see Fig. 4. The optical waveguide sample is immersed in a liquid of refractive index $n_{l}$ near the cladding refractive index $n_{\text {clad }}$ of the GRIN optical fibre or the refractive index of the substrate of the waveguide sample. An Abbe refractometer with an accuracy of \pm 0.0001 is used to measure the refractive indices of the immersion liquids. The collimated laser beam crosses the sample and passes through the microscope objective MO1 with magnification 10x and N.A. 0.25. An identical microscope objective $\mathrm{MO} 2$ is installed in the reference arm to eliminate the curvature of the 
optical field. The position of MO2 is precisely adjusted. These two beams are recombined at the beam splitter BS2, which is identical to BS1.

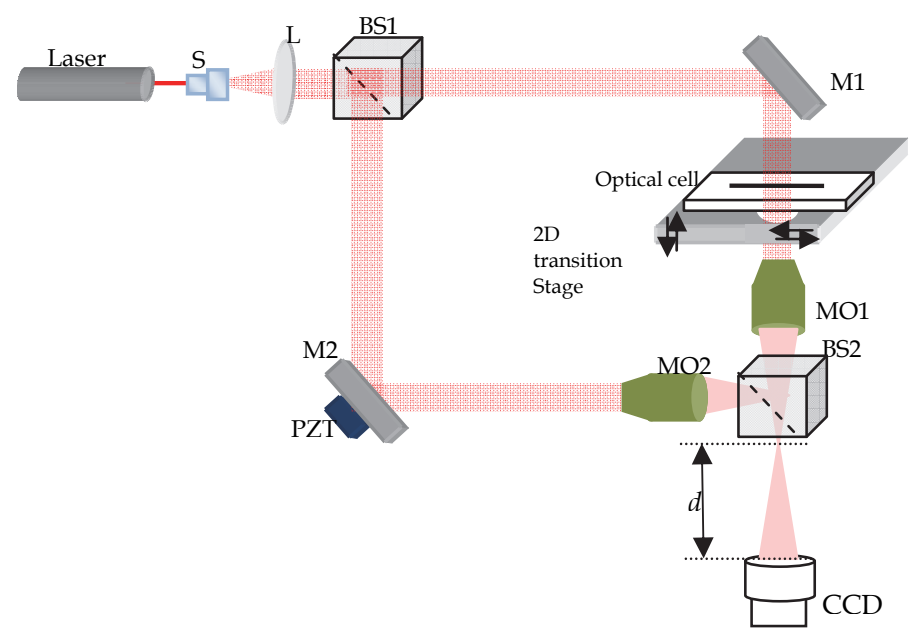

Fig. 4. Digital holographic interferometric set-up.

The mirror M2 in the digital holographic set-up reflects the reference beam. This mirror can be tilted to control the position of the reference beam on the CCD camera. The reference beam reaches the CCD camera at a small incident angle with respect to the propagation direction of the object wave. Therefore, the off axis holographic configuration is used (Wahba \& Kreis, 2009a, 2009b, 2009c; Yassien et al., 2010). In addition, the mirror M2 in the digital holographic setup is mounted on a piezoelectric transducer (PZT), which acts as the phase shifting tool. It is positioned in the reference arm and enables us to obtain four phase shifted holograms. The phase shifting steps start with $0^{\circ}$ and the holograms are shifted mutually by $\pi / 2$. These phase shifted holograms are recorded by an Allied Vision Marlin F145B2 CCD camera with pixel pitch $4.65 \mu \mathrm{m} \times 4.65 \mu \mathrm{m}$ and pixel numbers 1392, and 1040 in the horizontal and vertical directions, respectively.

\section{Results and discussion}

\subsection{Refractive index profile of GRIN optical waveguide}

Phase shifting digital holographic interferometry is used to investigate the optical parameters of GRIN optical waveguide samples. Fresnel off-axis holograms have been produced by using a Mach-Zehnder holographic arrangement; see Fig. 4. A piezoelectric transducer (PZT), acting as a phase-shifting tool, is applied in the reference arm and helps us to obtain four phase-shifted holograms. Fig. 5 represents the phase shifted digital holograms for a GRIN optical fibre sample. The GRIN optical fibre sample is immersed in a liquid of refractive index 1.46, which is greater than that of its cladding. The phase of the hologram (Fig. 5(a)) is assumed to be zero, whereas the phase shifts of the holograms (Fig. $5(\mathrm{~b})$ to $5(\mathrm{~d}))$ are $\pi / 2, \pi$, and $3 \pi / 2$; respectively. 


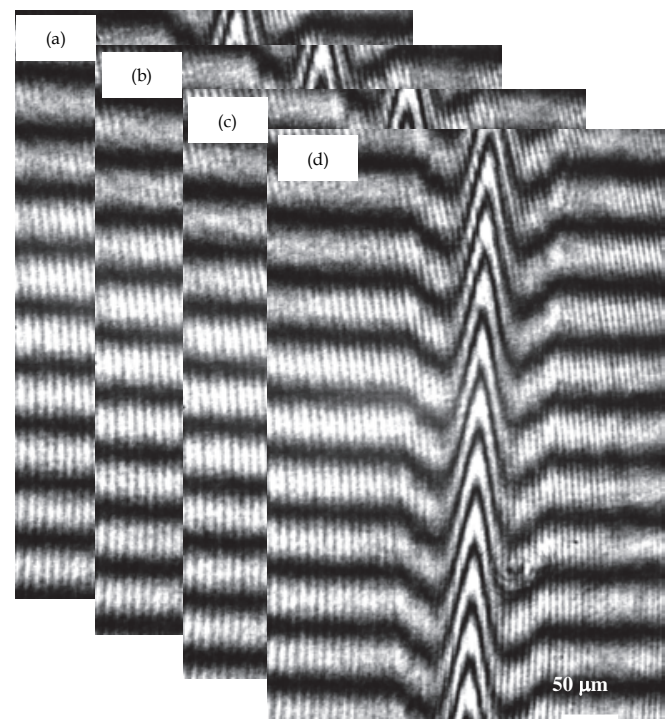

Fig. 5. Phase shifted digital holograms of the GRIN optical fibre sample at wavelength $\lambda=$ $632.8 \mathrm{~nm},(\mathrm{a}) 0,(\mathrm{~b}) \pi / 2$, (c) $\pi$, and (d) $3 \pi / 2$.

According to the phase shift algorithm, these four phase shifted holograms are used to produce the complex field in the hologram plane. From this field, the interference phase distribution is reconstructed by the convolution algorithm, and the result is shown in Fig. 6 . In this figure we have the wrapped interference phase. The phase has been unwrapped and then the linear increase of the background phase from left to right has been approximated by a linear regression and subtracted from the unwrapped interference phase. The result with normalized background is shown in Fig. 7(a). This image represents the interference phase map of the GRIN optical fibre sample, whose interference phase varies across the sample but remains nearly constant along the optical fibre sample. This is due to the fact that the refractive index of each layer of the optical fibre sample remains constant along the optical fibre sample. One notes that the GRIN optical fibre sample is tilted inside the optical phase map, see Fig. 7(a). The tilt angle of the sample is small but it increases the error in the calculated mean values of optical phase differences along the fibre. Also, it might cause a broadening of the calculated mean value of the fibre radius. A simple algorithm is used to avoid this tilting and its corresponding errors. The optical phase differences across the fibre are shifted in each row of the phase map matrix. The optical interference phase differences along each layer of the optical fibre are shifted to be in the same column of the phase map matrix. After the shifting of all rows, the optical interference phase differences along the fibre appear to be perpendicular to $x$-axis. Fig. $7(\mathrm{~b})$ represents the perpendicular optical phase differences along the GRIN optical fibre sample.

The modified interference optical phase map (Fig. 7(b)) is used to calculate the mean values of the interference optical phase differences across the GRIN optical fibre sample. Then the mean values of interference phase differences across the GRIN optical fibre in combination with the multilayer model are used to estimate the refractive index profile of the GRIN optical fibre. The refractive index profile of the GRIN optical fibre cladding is determined using the multilayer model. 


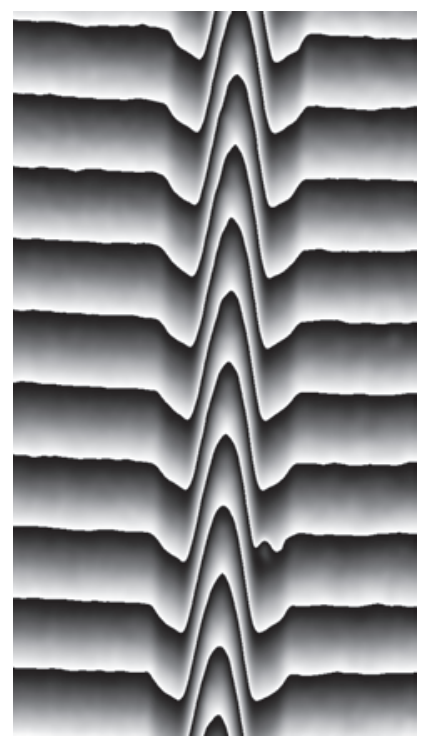

Fig. 6. Reconstructed interference phase modulo $2 \pi$ from the phase shifted digital holograms of Fig. 5.
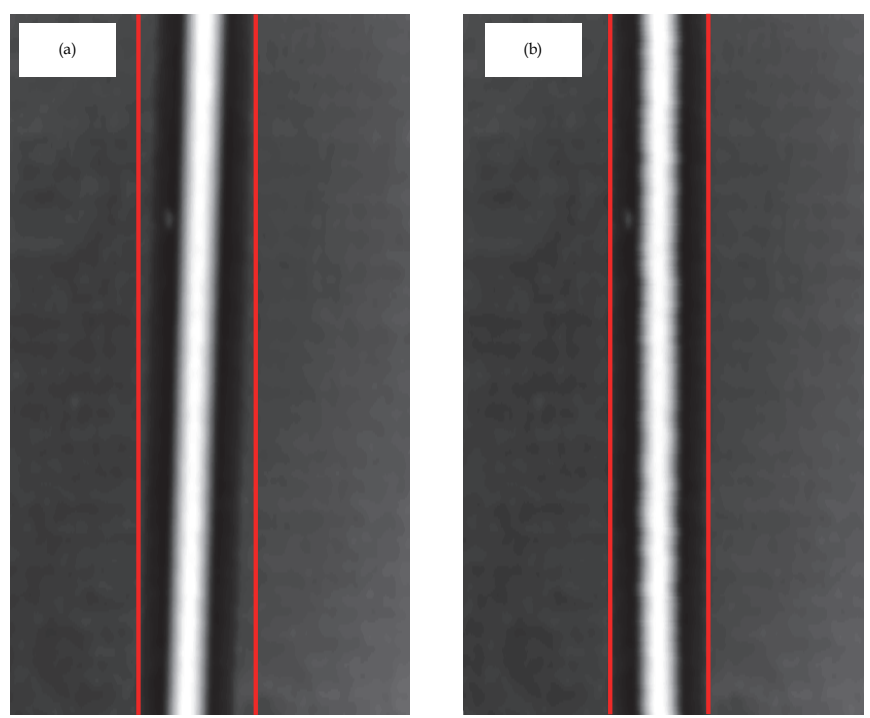

Fig. 7. Unwrapped interference phase distribution with normalized background of the GRIN optical fibre, (a) tilted and (b) perpendicular to $x$-axis.

Fig. 8 displays the calculated core refractive index profile of the GRIN optical fibre sample. These refractive indices are fitted using a parabolic function which could be used to determine the TE modes for the GRIN optical fibre. 


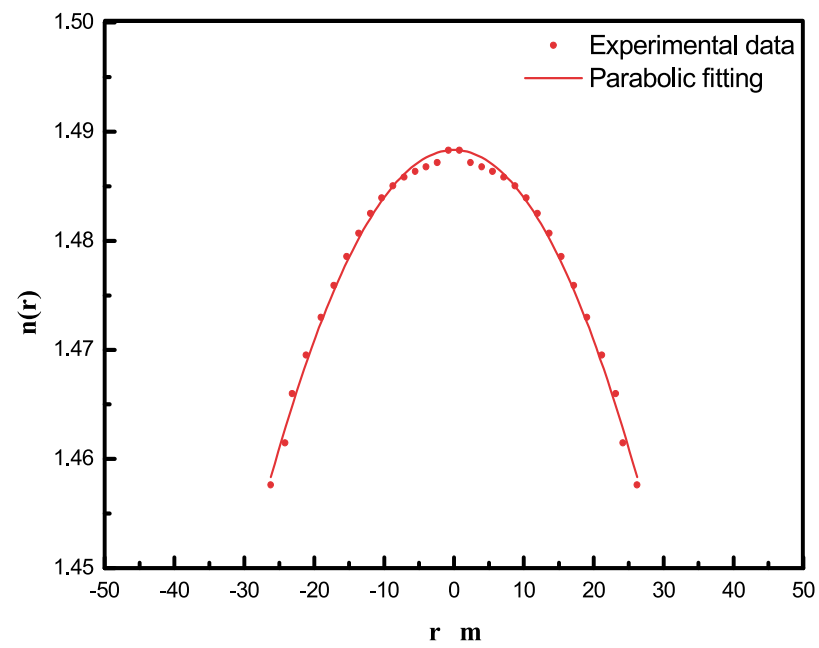

Fig. 8. The calculated refractive index profile of GRIN optical fibre.
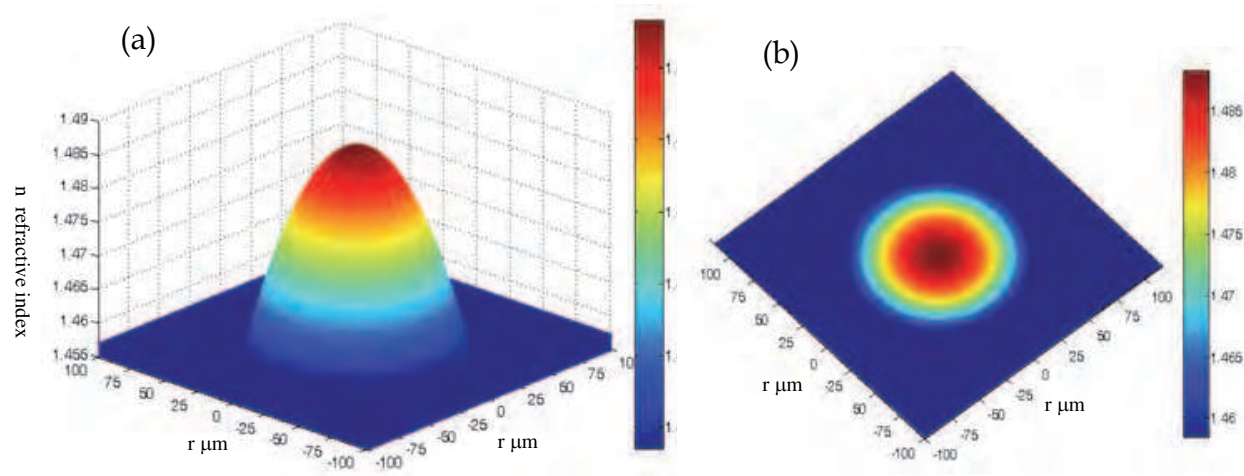

Fig. 9. (a) The reconstructed 3D refractive index profile, (b) the projection of the 3D refractive index profile of GRIN optical fibre.

According to the symmetry of GRIN optical fibre, where the GRIN optical fibre consists of a large number of coaxial layers, all points of each layer have the same refractive index. Whereas, the $3 \mathrm{D}$ refractive index can be presented in spherical polar coordinates as $n(r, \theta, \rho)=n_{0}+\Delta n(r / R)^{2}$ with $r=0: R, \theta=0: \pi$ and $\rho=0: 2 \pi$, then a simple algorithm based on the assumptions of the multilayer model is used to reconstruct the refractive index profile across the GRIN optical fibre. Hence, Fig. 9(a) shows the three dimensional view of the refractive index across the GRIN optical fibre. The projection of the three dimensional refractive index profile is presented in Fig. 9(b).

In addition the same procedures are applied to a fabricated GRIN optical waveguide. Hence the UV irradiation lithographic method is used to inscribe an optical waveguide in a slab of PMMA. The waveguide sample is prepared at $\Lambda=248 \mathrm{~nm}$, with laser pulse energy density $32 \mathrm{~mJ} / \mathrm{cm}^{2}, \mathrm{R}$ (repetition rate) $=5 \mathrm{~Hz}, \mathrm{~N}$ (number of pulses) $=1250$ pulse, and temperature 
of $22^{\circ} \mathrm{C}$. This sample is immersed in a fluid of $n_{L}=1.4917$ perfectly matching the refractive index of the substrate. The photo-induced refractive index variations in the waveguide area can be divided into two zones. The refractive index in the first zone is high at the top surface and decreases gradually until reaching a minimum value, so that the refractive index distribution in the first zone can be classified as a graded index profile. The refractive index distribution in the second zone looks like Gaussian shape. The original homogeneous material surrounds the modified area of the PMMA slab, where the refractive index of the PMMA slab is $n_{s}$. The cross sectional area of the waveguide is divided into $Q$ layers. The refractive index of each layer is constant. The refractive index of the $i^{\text {th }}$ layer is $n_{i}$, where $i=1,2, \ldots, Q$. In addition, the thickness of each layer is constant. The refractive index variations extend in the polymer slab up to depth $b$. The layer thickness is equal to $b / Q$.

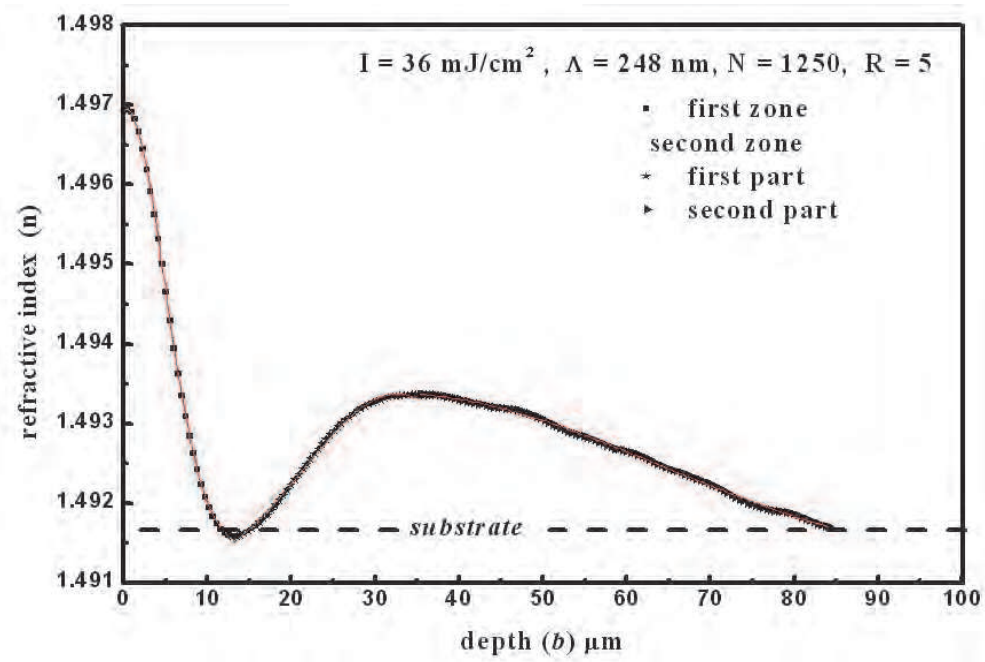

Fig. 10. The measured refractive index profile of fabricated GRIN optical waveguide.

The irradiated PMMA slab is immersed in a fluid and then it is used in the holographic setup. The object beam crosses the irradiated PMMA slab and then is superposed on reference beam. The produced interference pattern contains a field distribution related to the refractive index variations inside the modified area. The optical interference phase difference due to each layer depends on the refractive index $n_{i}$. The optical interference phase difference $\Delta \varphi_{i}$ across each layer is given by

$$
\frac{\Delta \varphi_{i}}{2 \pi} \lambda=\left(n_{i}-n_{s}\right) a_{i} \quad, i=1,2,3, \ldots \ldots . . Q
$$

where $a_{i}$ is the width of the $i^{\text {th }}$ layer of the waveguide, and $\lambda$ is the wavelength of the used light beam. The refractive index of PMMA slab (non-irradiated area) $n_{s}$ is equal to the refractive index of the immersion liquid $n_{L}$. This equation is valid in this case because the refractive index difference between the maximum and minimum values is very small in comparison with the refractive index difference of GRIN optical fibre. The calculated refractive index profile of the fabricted optical waveguide is shown in Fig.10. 


\subsection{Mode field distribution of GRIN optical waveguides}

The parabolic fitting of the refractive index profile for the investigated symmetric GRIN optical fibre core is shown in Fig. 8. The calculated optical parameters $n_{0}, \Delta n$ with their standard error $(S d)$ and $\Delta \varepsilon$ are presented in table 1. The accuracy of the measurements of these optical parameters using the digital holographic method is increased in comparison to the accuracy of the multiple-beam Fizeau fringes and the two-beam interference Pluta polarizing microscope (Hamza et al., 2001).

The corresponding parameters $\varepsilon_{\mathrm{o}}$ and $\varepsilon_{\mathrm{c}}$ for the centre of the core and the cladding of the optical waveguide respectively are determined. The solutions must be oscillatory in the core radius of the optical fibre in order to represent guided modes.

The modes are guided as long as the condition

$$
\sqrt{\varepsilon_{o}}>\frac{\beta}{k_{o}}>\sqrt{\varepsilon_{o}-\Delta \varepsilon}
$$

is satisfied.

The values of $\beta$ can be calculated directly by using Eq. (20) for every mode number $q$. The coefficient $A$ in Eq. (17) is related to the power carried in the core of the optical waveguide. The power is calculated by integrating the $z$ component of the time averaged Poynting vector over the cross-sectional area of the waveguide:

$$
S_{z}=-\frac{1}{2} \operatorname{Re}(E \times H \cdot \vec{z})
$$

The average power in TE modes is

$$
P_{z}=-\frac{1}{2} \int_{-\infty}^{\infty} E_{y} H_{x}^{*} d x=\frac{\beta}{2 \omega \mu_{0}} \int_{-\infty}^{\infty}\left|E_{y}\right|^{2} d x .
$$

The parameter $P$ does not indicate the total power but the power per unit length in the $y$ direction. The numbers of TE modes is related to the number of nodes, since the GRIN optical waveguide supports thirty nine modes, starting from $q=0$ to 38. Each propagated mode has an effective index given by Eq. (21).

\begin{tabular}{|l|l|l|l|}
\cline { 2 - 4 } \multicolumn{1}{c|}{} & $n_{0}$ & $\Delta n$ & $\Delta \varepsilon$ \\
\cline { 2 - 4 } \multicolumn{1}{c|}{} & 1.48824 & 0.02974 & 0.088526 \\
\hline$S d$ & $\pm 1.42441 \times 10^{-4}$ & $\pm 3.04453 \times 10^{-4}$ & \\
\hline
\end{tabular}

Table 1. The calculated optical parameters of GRIN optical fibre

Fig. 11 represents the effective indices for all modes of the GRIN optical waveguide sample. The values of the effective indices are confined between the core centre and clad refractive indices according to Eq. (21). 


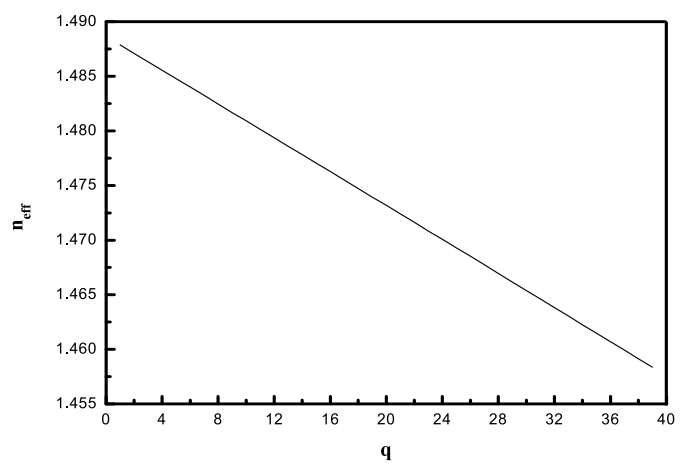

Fig. 11. Effective indices against the mode number $q$ for GRIN optical fibre sample.

Fig. 12 represents the first mode for the sample. Fig. 13 represents the second and the sixth modes. The oscillations are featured by an amplitude increasing with $x$ approaching $x_{t}^{i}$, where $i$ is the interface order between the modes. Landau and Lifshitz proved that the increase in wave amplitude, when the reflection point is reached, is a typical property in the presence of a varying index of refraction (Landau \& Lifshitz, 1960).

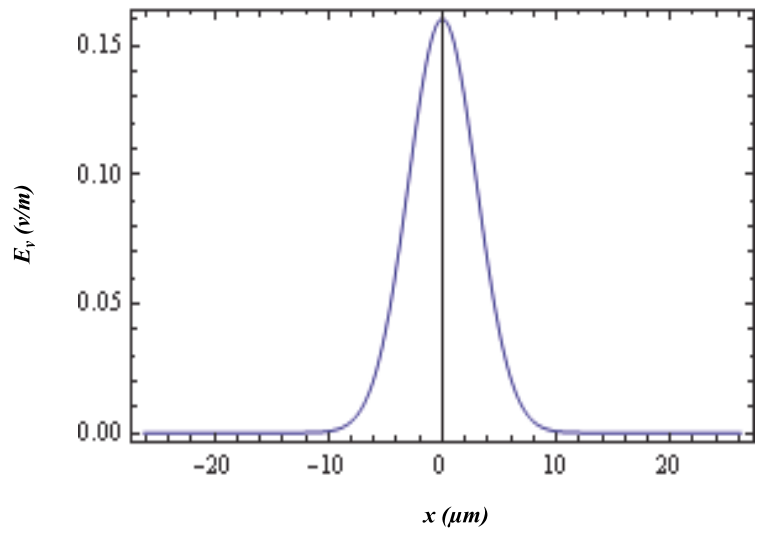

Fig. 12. Plot of the first mode $(q=0)$ calculated for GRIN optical waveguide sample.

Now we can calculate the effective indices $n_{\text {eff }}$ for each TE mode for an asymmetric (fabricated) optical waveguide. The guiding condition for the first zone is represented by

$$
n_{s 1}<\frac{\beta}{k_{o}}<n_{s 1}+\Delta n_{1}
$$

Also the guiding condition for the second zone, for the first and second parts are given respectively by

$$
n_{s 1}<\frac{\beta}{k_{o}}<n_{s 1}+\Delta n_{2}
$$


and

$$
n_{s}<\frac{\beta}{k_{o}}<n_{s}+\Delta n_{3}
$$

Eqs. (24), (25) and (26) can be used to calculate the effective indices $n_{\text {eff }}$ of each mode for every part of the second zone. The first zone of the sample supports three modes. The first zone of the effective index $n_{\text {eff }}$ for each mode is calculated. The numbers of modes in the first and second parts are four and eight modes respectively.

Generally, the number of the modes and the values of the effective indices $n_{\text {eff }}$ depend on two factors; the first one is the depth and the second one is $\Delta n$.

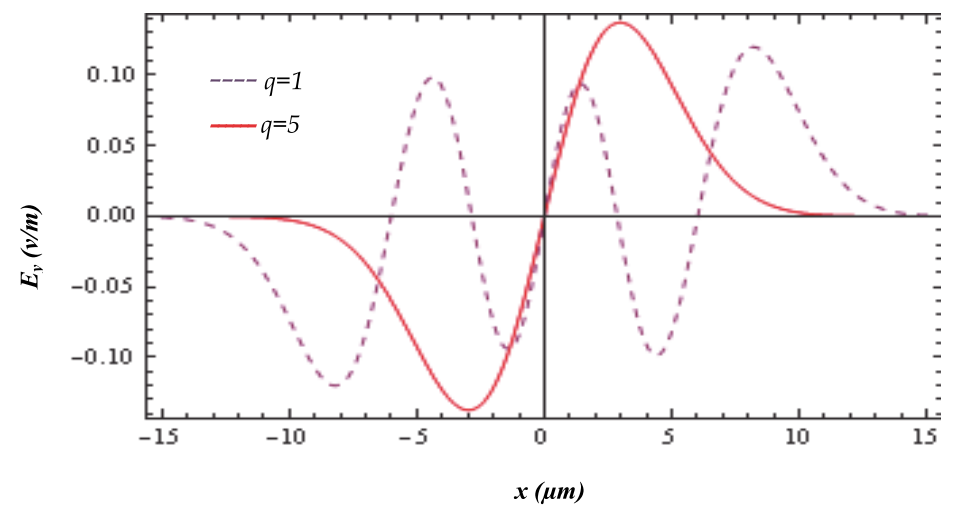

Fig. 13. Plots of the second mode $(q=1)$ and sixth mode $(q=5)$ calculated for GRIN optical waveguide sample.

\section{Conclusions}

In this chapter the digital holographic phase shifting interferometric method is used to investigate and characterize GRIN optical waveguide samples. Phase shifting digital holography has the advantages of no dc term and no twin image in the reconstructed field as well as high accuracy due to the redundant data in the multiple holograms. The optical interference phase differences due to the variation of refractive indices of GRIN optical waveguides are extracted. A simple algorithm is used to recover the tilting of the extracted optical interference phase differences along the GRIN optical wavegide, to avoid the errors of the calculated mean values of optical phase differences due to the GRIN optical waveguides. The broadening of optical waveguide radius or width is also reduced.

The advances of accurate reconstructed interference phase differences in combination of multilayer model are used to calculate the refractive index profiles of GRIN optical waveguide. Also, a simple algorithm based on the assumptions of the multilayer model is used to reconstruct the 3D refractive index profile of GRIN optical fibre.

The refractive index profiles of symmetric and asymmetric GRIN optical waveguides and their associated modes are calculated. The propagation coefficient $\beta$ and hence the effective indices $n_{\text {eff }}$ associated with every mode are determined. 


\section{Acknowledgment}

The authors would like to express their gratitude to Professor A A Hamza for his fruitful discussions and comments. In addition, the authors are greatly indebted to the Unit of Research Management of Mansoura University for financial support. We are also acknowledging the encouragement and support of the Vice president of Mansoura University for Higher studies \& Research."

\section{References}

Baker, A.K. \& Dyer, P.E. (1993). Refractive-index modification of polymethylmethacrylate (PMMA) thin films by KrF-laser irradiation, Appl. Phys. A, Vol. 57, pp. 543

Barakat, N., Hamza, A. A. \& Ganied, A. S. (1985). Multiple-beam interference fringes applied to GRIN optical waveguides to determine fiber characteristics, Appl. Opt., Vol. 24, pp. 4383-4386

Cha, S. S. \& Vest, C. M. (1981). Tomographic reconstruction of strongly refracting fields and its applications to interferometric measurements of boundary layers, Appl. Opt., Vol. 20, pp. 2787-2794

Chiang, K. S. (1985). Construction of refractive-index profiles of planar dielectric waveguides from the distribution of effective indexes, Journal of Lightwave Tech., Vol. 3, pp. 385

Colomb, T., Dahlgren, P., Beghuin, D., Cuche, E., Marquet, P. \& Depeursinge, C. (2002). Polarization Imaging by Use of Digital Holography, Appl. Opt., Vol. 41, pp. 27-37

Colomb, T., Dürr, F., Cuche, E., Marquet, P., Limberger, H. G., Salath, R. P. \& Depeursinge, C. (2005). Polarization microscopy by use of digital holography: application to optical-fiber birefringence measurements, Appl. Opt., Vol. 44, pp. 4461-4469

Conwell, e. m. (1973). Modes in optical waveguides formed by diffusion, Appl. Phys. Lett., Vol. 26, pp. 328

Cuche, E., Marquet, P. \& Depeursinge, C. (1999). Simultaneous amplitude-contrast and quantitative phase-contrast microscopy by numerical reconstruction of Fresnel offaxis holograms, Appl. Opt., Vol. 38, pp. 6994-7001

De Angelis, M., De Nicola, S., Finizio, A., Pierattini, G., Ferraro, P., Pelli, S., Righini, G. \& Sebastiani, S. (2006). Digital-holography refractive-index-profile measurement of phase gratings, Appl. Phys. Lett., Vol. 88, pp. 111-114

Dubois, F., Joannes, L., Dupont, O., Dewandel, J. L. \& Legros, J. C. (1999). An integrated optical set-up for fluid-physics experiments under microgravity conditions, Meas. Sci. Technol., Vol. 10, pp. 934-945

Eguchi, M. \& Horinouchi, S. (2004). Finite-Element Modal Analysis of Large-Core Multimode Optical Fibers, Appl. Opt., Vol. 43, pp. 2163-2167

Eldada, L. \& Shacklette, L. W. (2000). Advances in polymer integrated optics, IEEE J. Sel. Top. Quantum Electron., Vol. 6 (1), pp. 54-68

El-Morsy, M. A., Yatagai, T., Hamza, A., Mabrouk, M. A. \& Sokkar, T. Z. N. (2002a). Multiple-beam Fizeau fringe-pattern analysis using Fourier transform method for accurate measurement of fiber refractive index profile of polymer fiber, J. Appl. Polymer Sci., Vol. 85, pp. 475-484 
El-Morsy, M. A., Yatagai, T., Hamza, A., Mabrouk, M. A. \& Sokkar, T. Z. N. (2002b). Automatic refractive index profiling of fibers by phase analysis method using Fourier transform, Opt. and Lasers in Eng., Vol. 38, pp. 509-525

Faust, R. C. (1952).An Interferometric Method of Studying Local Variations in the Refractive Indices of a Solid, Proc. Phys. Soc. B, Vol. 65, pp. 48-62

Faust, R. C. (1954).The Determination of the Refractive Indices of inhomogeneous Solids by Interference Microscopy, Proc. Phys. Soc. B, Vol. 67, pp. 138-148

Gabor, D. (1948). A new microscopic principle, Nature, Vol. 161, pp. 777-778

Gabor, D. (1949). Microscopy by reconstructed wavefronts, Proc. Royal Society, A 197, pp. 454-487

Gabor, D. (1951). Microscopy by reconstructed wavefronts: II, Proc. Royal Society, B 64, pp. $449-469$

Hamza, A. A. , Sokkar, T.Z.N., Mabrouk, M. A., Ghandar, A. M. \& Ramadan, W. A. (1994). On the determination of the refractive index of a fibre: I. Skin-core fibre, Pure Appl. Opt., Vol. 3, pp. 943-961

Hamza, A. A., Ghander, A. M., Sokkar, T. Z. N., Mabrouk, M. A. \& Ramadan, W. A. (1995). On the determination of the refractive index of a fibre II. Graded index fibre ,Pure Appl. Opt., Vol. 4, pp. 161-177

Hamza, A. A., Mabrouk, M. A., Ramadan, W. A. \& Shams-Eldin, M. A. (2001). Determination of GRIN optical fibre parameters from transverse interferograms considering the refraction of the incident ray by the fibre, Opt. Commun., Vol. 200, pp. 131-138

Hamza, A.A., Belal, A.E., Sokkar, T.Z.N., EL-Dessouky, H.M. \& Agour, M.A. (2007). Interferometric studies on the influence of temperature on the optical and dispersion parameters of GRIN optical fibre, Opt. and Lasers in Eng., Vol. 45, pp. 145-152

Hamza, A.A., Mabrouk, M.A., Ramadan, W.A. \& Wahba, H.H. (2004). Core-index determination of a thick fibre using lens-fibre interference (LFI) technique, Opt. and Lasers in Eng., Vol. 42, pp. 121-130

Hariharan, P. (2002). Basics Of Holography, Cambridge University Press, ISBN 0521807417, Cambridge, United Kingdom

Hariharan, P., Oreb, B. F. \& Brown, N. (1982). A digital phase-measurement system for realtime holographic interferometry, Opt. Commun., Vol. 41, pp. 393-396

Hossain, M. M., Mehta, D. S. \& Shakher, C. (2006). Refractive index determination: an application of lensless Fourier digital holography, Opt. Eng., Vol. 45, pp. 106203

Kebbel, V., Adams, M., Hartmann, H. J. \& Jüptner, W. (1999). Digital holography as a versatile optical diagnostic method for microgravity experiments, Meas. Sci. Technol., Vol. 10, pp. 893-899

Kemper, B. \& Carl, D. (2006). Investigation of living pancreas tumor cells by digital holographic microscopy , J. Biomed. Opt., Vol. 11, pp. 034005

Kemper, B., Kosmeier, S., Langehanenberg, P. \& Bally, G. (2007). Integral refractive index determination of living suspension cells by multifocus digital holographic phase contrast microscopy, J. Biomed. Opt., Vol. 12, pp. 054009

Kreis, Th. (1996). Holographic Interferometry: Principles and Methods, Akademie-Verlag, ISBN 30-05-501644-0, Berlin, Germany 
Kreis, Th. (2005). Handbook of Holographic Interferometry, Optical and Digital Methods, WileyVCH, ISBN: 3-527-40546-1, Weinheim, Germany

Landau, L. D. and Lifshitz, E. M. (1960). Electrodynamics of Continuous Media, Pergamon, New York, USA

Marcuse, D. (1973). TE modes of graded-index slab waveguides, IEEE J. Quantum Electron, Vol. 9, pp. 1000

Marhic, M. E., Ho, P. S. \& Epstein, M. (1975). Nondestructive refractive-index profile measurements of clad optical fibers, Appl. Phys. Lett., Vol. 26, pp. 574-575

Mathey, P. \& Jullien, P. (1996). Numerical analysis of a WKB inverse method in view of index profile reconstruction in diffused waveguides, Opt. Commun., Vol. 122, pp. 127

Mathey, P., and Jullien, P. (1996). Numerical analysis of a WKB inverse method in view of index profile reconstruction in diffused waveguides, Opt. Commun., Vol. 122, pp. 127

Owen, R. B. \& Zozulya, A. A. (2002). Comparative Study with Double-Exposure Digital Holographic Interferometry and a Shack-Hartmann Sensor to Characterize Transparent Material, Appl. Opt., Vol. 41, pp. 5891-5895

Powell, R. L. \& Stetson, K. A. (1965).Interferometric vibration analysis by wavefront reconstruction, J. Opt. Soc. Am., Vol. 55, pp. 1593-1608

Rappaz, B., Marquet, P., Cuche, E., Emery, Y., Depeursinge, C. \& Magistretti, P. (2005). Measurement of the integral refractive index and dynamic cell morphometry of living cells with digital holographic microscopy, Opt. Exp., Vol. 13, pp. 9361-9373

Saunder, M. J. \& Gardner, W. B. (1977). Nondestructive interferometric measurement of the delta and alpha of clad optical fibers, Appl. Opt., Vol. 16, pp. 2368- 2371

Schnars, U. \& Jüptner, W. (1994a). Direct recording of holograms by a CCD target and numerical reconstruction, Appl. Opt., Vol. 33, pp. 179-181

Schnars, U. \& Jüptner, W. (1994b). Digital recording and reconstruction of holograms in hologram interferometry and shearography, Appl. Opt., Vol. 33, pp.4373-4377

Schnars, U. \& Jüptner, W. (2005).Digital holography: digital hologram recording, numerical reconstruction, and related techniques, Published by Springer, ISBN 3-540-21934-X , Germany

Schnars, U. (1994). Direct phase determination in hologram interferometry with use of digitally recorded holograms, J. Opt. Soc. Amer. A, Vol. 11, pp. 2011-2015

Schnars, U., Kreis, Th. \& Jüptner, W. (1995).Digital recording and numerical reconstruction of holograms: Reduction of the spatial frequency spectrum, Opt. Eng., Vol. 35, pp. 977-982

Shams-Eldin, M.A. , Wochnowski, C., Metev, S., Hamza, A.A. and Jüptner, W. (2004). Determination of the refractive index depth profile of an UV-laser generated waveguide in a planar polymer chip, Appl. Surf. Sci., Vol. 236, pp. 31

Shams-Eldin, M.A., Wochnowski, C., Koerdt, M., Metev, S., Hamza, A.A. and Jüptner, W. (2005). Characterisation of the optical-functional properties of a waveguide written by an UV-laser into a planar polymer chip, Opt. Mater., Vol. 27, pp.1138

Shemirani, M.B. \& Kahn, J.M. (2009). Higher-Order Modal Dispersion in Graded-Index Multimode Fiber, J. Lightwave Tech., Vol. 27, 5461 
Shemirani, M.B., Wei Mao Panicker, R.A. \& Kahn, J.M. (2009). Principal Modes in GradedIndex Multimode Fiber in Presence of Spatial- and Polarization-Mode Coupling, J. Lightwave Tech., Vol. 27, pp. 1248

Skarman, B.,Becker, J. \&Wozniak, K. (1996). Simultaneous 3D-PIV and temperature measurements using a new CCD-based holographic interferometer, Flow Meas. Instrum., Vol. 7, pp. 1-6

Sokkar, T. Z. N., El-Morsy, M. A. \& Wahba, H. H. (2008). Automatic fringe analysis of the induced anisotropy of bent optical fibres, Opt. Commun., Vol. 281, pp. 1915-1923

Sweeney, D. W. \& Vest, C. M. (1973). Reconstruction of three dimensional refractive index fields from multidirectional interferometric data, Appl. Opt., Vol. 12, pp. 2649-2663

Vollertsen, F. \& Wochnowski, C. (2004). UV-laser assisted Fabrication of integrated-optical Waveguides, CIRP Annals - Manufacturing Technology, Vol. 53, pp. 199-202

Wahba, H. H. \& Kreis, Th. (2009c). Digital holographic interferometric characterization of bent optical fibers, J. Opt. A: Pure Appl. Opt., Vol. 11, pp.105407

Wahba, H. H. \& Kreis, Th. (2009a). Characterization of graded index optical fibers by digital holographic interferometry, Appl. Opt., Vol. 48, pp. 1573-1582

Wahba, H. H. \& Kreis, Th. (2009b). Characterization of optical fibers by digital holographic interferometry", Proc. SPIE 7389, 73890K, Munich, Germany, June, 2009

Wochnowski, C. , Metev, S. and Sepold, G.(2000). UV-laser-assisted Modification of the optical Properties of Polymethylmethacrylate,"Appl. Surf. Sci., Vol.154-155, pp. 706-711

Yamaguchi, I. \& Zhang, T. (1997). Phase-shifting digital holography, Opt. Lett., Vol. 22, pp. 1268-1270

Yassien, K., Agour, M., V. Koyplow, C. \& Dessouky, H. (2010). On the Digital Holographic Interferometry of Fibrous Materials, I: Optical Properties of Polymer and Optical Fibers, Opt. and Lasers Eng., Vol. 48, pp.555 
ADVANCED HOLOGRAPHY METROLOGY AND IMAGING

Edace by trabele Napdanowa

\section{Advanced Holography - Metrology and Imaging \\ Edited by Dr Izabela Naydenova}

ISBN 978-953-307-729-1

Hard cover, 374 pages

Publisher InTech

Published online 09, November, 2011

Published in print edition November, 2011

Advanced Holography - Metrology and Imaging covers digital holographic microscopy and interferometry, including interferometry in the infra red. Other topics include synthetic imaging, the use of reflective spatial light modulators for writing dynamic holograms and image display using holographic screens. Holography is discussed as a vehicle for artistic expression and the use of software for the acquisition of skills in optics and holography is also presented. Each chapter provides a comprehensive introduction to a specific topic, with a survey of developments to date.

\section{How to reference}

In order to correctly reference this scholarly work, feel free to copy and paste the following:

Hamdy Wahba and Mamdouh Shams El-Din (2011). Digital Holographic Interferometric Characterization of Optical Waveguides, Advanced Holography - Metrology and Imaging, Dr Izabela Naydenova (Ed.), ISBN: 978953-307-729-1, InTech, Available from: http://www.intechopen.com/books/advanced-holography-metrologyand-imaging/digital-holographic-interferometric-characterization-of-optical-waveguides

\section{INTECH}

open science | open minds

\section{InTech Europe}

University Campus STeP Ri

Slavka Krautzeka 83/A

51000 Rijeka, Croatia

Phone: +385 (51) 770447

Fax: +385 (51) 686166

www.intechopen.com

\section{InTech China}

Unit 405, Office Block, Hotel Equatorial Shanghai

No.65, Yan An Road (West), Shanghai, 200040, China

中国上海市延安西路65号上海国际贵都大饭店办公楼 405 单元

Phone: +86-21-62489820

Fax: +86-21-62489821 
(C) 2011 The Author(s). Licensee IntechOpen. This is an open access article distributed under the terms of the Creative Commons Attribution 3.0 License, which permits unrestricted use, distribution, and reproduction in any medium, provided the original work is properly cited. 